\title{
The impact of cobalt-60 source age on biologically effective dose in high-dose functional Gamma Knife radiosurgery
}

\author{
Benjamin H. Kann, MD, ${ }^{1}$ James B. Yu, MD, MHS, ${ }^{1}$ John M. Stahl, MD, ${ }^{1}$ James E. Bond, PhD, ${ }^{1}$ \\ Christopher Loiselle, MD, ${ }^{2}$ Veronica L. Chiang, MD, ${ }^{3}$ Ranjit S. Bindra, MD, PhD, ${ }^{1}$ \\ Jason L. Gerrard, MD, PhD, ${ }^{3}$ and David J. Carlson, PhD' \\ Departments of ${ }^{1}$ Therapeutic Radiology and ${ }^{3}$ Neurosurgery, Yale University School of Medicine, New Haven, Connecticut; and \\ 2Department of Radiation Oncology, Swedish Cancer Institute, Seattle, Washington
}

\begin{abstract}
OBJECTIVE Functional Gamma Knife radiosurgery (GKRS) procedures have been increasingly used for treating patients with tremor, trigeminal neuralgia (TN), and refractory obsessive-compulsive disorder. Although its rates of toxicity are low, GKRS has been associated with some, if low, risks for serious sequelae, including hemiparesis and even death. Anecdotal reports have suggested that even with a standardized prescription dose, rates of functional GKRS toxicity increase after replacement of an old cobalt-60 source with a new source. Dose rate changes over the course of the useful lifespan of cobalt-60 are not routinely considered in the study of patients treated with functional GKRS, but these changes may be associated with significant variation in the biologically effective dose (BED) delivered to neural tissue.

METHODS The authors constructed a linear-quadratic model of BED in functional GKRS with a dose-protraction factor to correct for intrafraction DNA-damage repair and used standard single-fraction doses for trigeminal nerve ablation for TN (85 Gy), thalamotomy for tremor (130 Gy), and capsulotomy for obsessive-compulsive disorder (180 Gy). Dose rate and treatment time for functional GKRS involving 4-mm collimators were derived from calibrations in the authors' department and from the cobalt-60 decay rate. Biologically plausible values for the ratio for radiosensitivity to fraction size $(\alpha / \beta)$ and double-strand break (DSB) DNA repair halftimes $(\tau)$ were estimated from published experimental data. The biphasic characteristics of DSB repair in normal tissue were accounted for in deriving an effective $\tau_{1}$ halftime (fast repair) and $\tau_{2}$ halftime (slow repair). A sensitivity analysis was performed with a range of plausible parameter values.
\end{abstract}

RESULTS After replacement of the cobalt-60 source, the functional GKRS dose rate rose from 1.48 to $2.99 \mathrm{~Gy} / \mathrm{min}$, treatment time fell, and estimated BED increased. Assuming the most biologically plausible parameters, source replacement resulted in an immediate relative BED increase of $11.7 \%$ for GKRS-based TN management with 85 Gy, $15.6 \%$ for thalamotomy with $130 \mathrm{~Gy}$, and $18.6 \%$ for capsulotomy with $180 \mathrm{~Gy}$. Over the course of the 63 -month lifespan of the cobalt-60 source, BED decreased annually by $2.2 \%$ for TN management, $3.0 \%$ for thalamotomy, and $3.5 \%$ for capsulotomy.

CONCLUSIONS Use of a new cobalt-60 source after replacement of an old source substantially increases the predicted BED for functional GKRS treatments for the same physical dose prescription. Source age, dose rate, and treatment time should be considered in the study of outcomes after high-dose functional GKRS treatments. Animal and clinical studies are needed to determine how this potential change in BED contributes to GKRS toxicity and whether technical adjustments should be made to reduce dose rates or prescription doses with newer cobalt-60 sources.

http://thejns.org/doi/abs/10.3171/2016.6.GKS161497

KEY WORDS Gamma Knife; functional radiosurgery; biologically effective dose; thalamotomy; trigeminal neuralgia; capsulotomy; stereotactic radiosurgery

ABBREVIATIONS BED = biologically effective dose; $D S B=$ double-strand break; GKRS = Gamma Knife radiosurgery; $L Q=$ linear-quadratic; $T N=$ trigeminal neuralgia. SUBMITTED June 8, 2016. ACCEPTED June 30, 2016.

INCLUDE WHEN CITING DOI: 10.3171/2016.6.GKS161497. 
$\mathrm{O}$ VER the past several decades, use of Gamma Knife radiosurgery (GKRS) for managing functional neurological disorders has become more common. First applied in the management of trigeminal neuralgia (TN), functional GKRS has since been used for treating patients with essential, parkinsonian, and multiple sclerosis-related tremor; movement disorders; obsessivecompulsive disorder; and medically refractory aggressiveness. ${ }^{6}$ In these interventions, functional radiosurgery typically consists of a 1-time, high-dose treatment to a predefined, specific neuroanatomical location, involving a single GKRS shot with a 4-mm collimator. ${ }^{12,14,22}$ The doses used in functional GKRS typically range from 80 to 180 Gy and therefore are much higher than those used in the treatment of patients with malignant or benign tumors. Although functional GKRS has been reported to have low rates of toxicity in most series, it has, in rare instances, been associated with severe neurological sequelae and even death from radionecrosis. ${ }^{6,19,22,27}$ Given that functional GKRS is used to improve the quality of life of patients with functional disorders, it is of utmost importance to investigate factors that may contribute to treatment-related adverse effects in GKRS.

Although there have been studies supporting an association of functional GKRS parameters such as prescription dose, target location, and treatment volume with toxicity, ${ }^{20,24}$ the impact of the age of the cobalt- 60 source has been seldom studied. Cobalt- 60 source age directly correlates with dose rate and treatment time, factors that may independently influence the biologically effective dose (BED) of treatment depending on the propensity for the target tissue to undergo intrafraction, sublethal, doublestrand break (DSB) DNA-damage repair. ${ }^{11,18}$ The target tissues for functional GKRS, normal CNS tissues, have a greater innate potential for DNA repair than tumor tissues do, a fact that may be more important to consider in functional GKRS than in GKRS-based tumor management. ${ }^{11}$ Furthermore, a cobalt-60 source may age significantly over the course of its use before replacement, resulting in large variations in dose rate and treatment times in functional GKRS procedures.

Very few studies have analyzed the relationship between the age of the cobalt-60 source and clinical end points, and to our knowledge, no studies on functional GKRS techniques have modeled BED over the course of the cobalt-60 effective lifespan. Anecdotally, we had observed an increase in adverse events after GKRS-based thalamotomy following source replacement, an observation that motivated the present investigation. Given the uniquely high doses and long treatment times required in functional GKRS, we sought to investigate the potential radiobiological impact of cobalt-60 source age on BED in functional GKRS procedures.

\section{Methods}

\section{Model Construction}

We constructed a radiobiological model based on the linear-quadratic (LQ) model, ,16 with corrections for intrafraction DNA repair. ${ }^{23}$ The model included the doseprotraction factor to estimate the BED to the tissue at the
TABLE 1. Literature summary of DNA repair halftime and $\boldsymbol{\alpha} / \boldsymbol{\beta}$ values derived from experimental data for rat spinal cord

\begin{tabular}{lcccc}
\hline Authors \& Year & $\begin{array}{c}\text { Fast } \\
\text { Halftime } \\
\tau_{1}(\mathrm{hrs})\end{array}$ & $\begin{array}{c}\text { Slow } \\
\text { Halftime } \\
\tau_{2} \text { (hrs) }\end{array}$ & $\begin{array}{c}\text { Proportional } \\
\text { Fast Halftime } \\
\text { Repair (hrs) }\end{array}$ & $\begin{array}{c}\alpha / \beta \\
(\mathrm{Gy})\end{array}$ \\
\hline Ang et al., 1992 & 0.70 & 3.80 & 0.38 & 2.0 \\
\hline Landuyt et al., 1997 & 0.25 & 6.40 & 0.50 & 2.0 \\
\hline Pop et al., 2000 & 0.19 & 2.16 & 0.51 & 2.5 \\
\hline
\end{tabular}

target isocenter with prescription doses representative of 3 common functional GKRS treatments: 180 Gy (in patients undergoing ventral capsulotomy for obsessive-compulsive disorder), $130 \mathrm{~Gy}$ (in patients undergoing thalamotomy for tremor), and 85 Gy (in patients undergoing trigeminal nerve root ablation for TN). The BED for prolonged exposures at low dose rates can be calculated with the following equation:

$$
\mathrm{BED}=n^{*} d\left[1+\frac{G[\lambda, \mathrm{T}] * d}{\alpha / \beta}\right]
$$

where $n$ is the number fractions delivered to the tissue, $\alpha / \beta$ the ratio for radiosensitivity to fraction size, and $d$ the prescribed dose (in Gy) per fraction; the "dose-protraction factor $G$ " is defined as

$$
\begin{aligned}
& G=\frac{2\left(e^{-\lambda T}+\lambda T-1\right)}{(\lambda T)^{2}} \\
& \lambda=\frac{\ln 2}{\tau}
\end{aligned}
$$

where $T$ is the radiation treatment time, and $\tau$ is the DSB DNA repair halftime intrinsic to the irradiated tissue. ${ }^{23}$ The protraction factor, $G$, quantifies intrafraction DSB DNA repair of radiation-induced damage during continuous irradiation.

\section{Model Parameters and Assumptions}

Calculations were performed for 3 single-fraction prescription doses of 180, 130, and 85 Gy $(n=1$ in Eq. 1 for single-fraction functional GKRS). The radiation treatment time $T$ was derived from the cobalt-60 source age, initial source dose rate, and the exponential decay function for cobalt-60 with a source half-life of approximately 5.27 years (i.e., 63 months). The initial source dose rate for a single GKRS shot with a 4-mm collimator at $2.99 \mathrm{~Gy} / \mathrm{min}$ was derived from institutional source calibration.

Extensive study of DNA repair of radiation-induced damage in rat spinal cord has shown that the rate of DNA repair is not monoexponential but probably at least biexponential in nature. Multiple studies have elucidated a biphasic rate of DSB DNA repair and have derived 2 repair halftimes: a fast repair time $\left(\tau_{1}\right)$ lasting generally less than 1 hour and a slow repair time $\left(\tau_{2}\right)$ lasting $2-6$ hours. ${ }^{1,15,21}$ These studies are summarized in Table 1 . The protraction factor $G$ was derived by calculating protraction factors for each $\tau_{1}$ and $\tau_{2}$ and calculating a weighted average of the 2 
TABLE 2. Treatment times, absolute BEDs, and changes in BED of functional GKRS after cobalt-60 source replacement

\begin{tabular}{lccccccc}
\hline \multirow{2}{*}{ Procedure } & \multicolumn{2}{c}{ Treatment Time (mins) } & & \multicolumn{2}{c}{ BED* in Gy (range) } & \% BED Change w/ \\
\cline { 2 - 3 } & Old Source† & New Source & & Old Source $\dagger$ & New Source & & New Source (range) \\
\hline TN ablation w/ 85 Gy & 57 & 28 & & $2888(2466-3179)$ & $3225(2899-3415)$ & $11.7(7.4-17.5)$ \\
\hline Thalamotomy w/ 130 Gy & 88 & 43 & & $6087(5050-6891)$ & $7034(6119-7619)$ & $15.6(10.6-21.2)$ \\
\hline Capsulotomy w/ 180 Gy & 121 & 60 & & $10,652(8668-12,306)$ & $12,632(10,724-13,965)$ & $18.6(13.5-23.7)$ \\
\hline
\end{tabular}

* Represents BED at isocenter; BED values are for most plausible $\tau$ values ( $\tau_{1}=0.38$ hours and $\tau_{2}=4.1$ hours), with the ranges indicating shortest to longest plausible $\tau_{1}$ and $\tau_{2}$ values.

$\dagger$ Old source represents cobalt-60 source with an initial dose rate of 2.99 Gy after 63 months of decay.

calculated protraction factors. A weighting of 50:50 was used as an approximation of the literature values. ${ }^{1,15,21}$

We conducted several sensitivity analyses for the model with the $\tau_{1}(0.19-0.70$ hours $)$ and $\tau_{2}(2.16-6.40$ hours $)$ DNA repair halftime values reported in the literature. An $\alpha / \beta$ value of 2 Gy was used to reflect the value commonly used for normal CNS tissue. We then calculated biologically most plausible estimates by using an average of the literature values (i.e., $\tau_{1} 0.38$ hours, $\tau_{2} 4.1$ hours, and $\alpha / \beta$ value 2 Gy). We estimated the impact of cobalt- 60 source replacement on dose rate, treatment time, and BED by assuming a cobalt-60 lifespan of 1 half-life (63 months) before replacement. Sensitivity analyses with $\alpha / \beta$ values ranging from 2 to $10 \mathrm{~Gy}$ were also performed. For these sensitivity analyses, we again used the aforementioned biologically most plausible estimates of DNA repair halftimes.

\section{Results}

With increasing cobalt-60 age, dose rate decreased and treatment time increased for each high-dose functional GKRS procedure. Source replacement after 63 months resulted in a doubling of the dose rate from 1.48 to $2.99 \mathrm{~Gy} /$ min and in an associated halving of treatment times for each procedure (Table 2). Treatment time decreased from 57 to 28 minutes for TN management with $85 \mathrm{~Gy}, 88$ to 43 minutes for thalamotomy with $130 \mathrm{~Gy}$, and 121 to 60 minutes for capsulotomy with $180 \mathrm{~Gy}$.

The estimated BED decreased for each GKRS procedure with increasing cobalt-60 source age, and the magnitude of this decrease depended on the assumption of the length of the DNA repair halftime (Fig. 1). Using the biologically most plausible assumptions for DNA repair halftimes (i.e., $\tau_{1} 0.38$ hours and $\tau_{2} 4.1$ hours), we noted that replacement of cobalt- 60 with a new source resulted in absolute and relative BED increases for each procedure (Table 2). In our model, source replacement resulted in immediate relative increases of BED by $11.7 \%$ for GKRSbased TN management with 85 Gy, $15.6 \%$ for thalamotomy with $130 \mathrm{~Gy}$, and $18.6 \%$ for capsulotomy with $180 \mathrm{~Gy}$. Varying the assumptions for $\tau$ over plausible DNA repair halftimes resulted in ranges of BED increase of 7.4\%$17.5 \%$ for TN management, $10.6 \%-21.2 \%$ for thalamotomy, and $13.5 \%-23.7 \%$ for capsulotomy. Averaged over the 63-month source lifespan of the cobalt-60 source, the BED decreased annually by $2.2 \%$ for $\mathrm{TN}$ management, $3.0 \%$ for thalamotomy, and $3.5 \%$ for capsulotomy. Testing our model with $\alpha / \beta$ values ranging from 2 to $10 \mathrm{~Gy}$ resulted in only negligible changes to BED (Fig. 2).

\section{Discussion}

Using an LQ-based radiobiological model, we have shown that despite the use of standardized prescription doses, cobalt-60 source age potentially causes substantial variation in BED during several high-dose functional GKRS procedures. Furthermore, our model also predicted that source replacement substantially and immediately increases BED by approximately $10 \%-20 \%$, followed by a decline annually by $2 \%-3 \%$ per year. The predicted BED increase associated with cobalt-60 source replacement may increase the risk for treatment-related toxicity and highlights that cobalt- 60 source age should be taken into consideration in future studies of functional GKRS treatments.

It has been well established that the radiation dose rate affects BED, but this effect is generally neglected in the planning of GKRS treatments. The magnitude of the BED change estimated by the model was highly dependent on the time it takes for the target tissue to undergo intrafraction DNA repair. Assuming that intrafraction DNA repair is negligible (i.e., that DNA repair halftimes are much longer than irradiation time), one would expect that cobalt-60 source age has little effect on the BED. In most cases, GKRS is used in practice for tumor management, in which relatively lower radiation doses of generally less than 30 Gy are used. Given these lower doses and shorter treatment times, it is plausible that variation in cobalt-60 source age would be less important in tumor management, because in GKRS for tumors, irradiation time is generally shorter than the time required for significant DNA repair in the target tissue. In contrast, functional GKRS treatments can take hours, and the length of irradiation time can vary significantly, depending on the age of the radiation source. The findings in the current study suggest that, given the existing literature on DNA repair times, cobalt-60 source age variation may be clinically meaningful for high-dose functional GKRS. As the results with the model indicated, the higher the prescription dose (and the longer the treatment time), the stronger the correlation between source age and BED.

By comparison, BED was much less sensitive to changes in the $\alpha / \beta$ value. Increasing $\alpha / \beta$ in the model from 2 to 10 Gy resulted in only very small decreases in the magnitude of BED change with source age. Assuming high $\alpha / \beta$ values, and thus less sensitivity to fraction size, we noted that source age had a weaker correlation with BED. There are many other factors that contribute to functional GKRS toxicity, including the procedure type, patient-specific fac- 

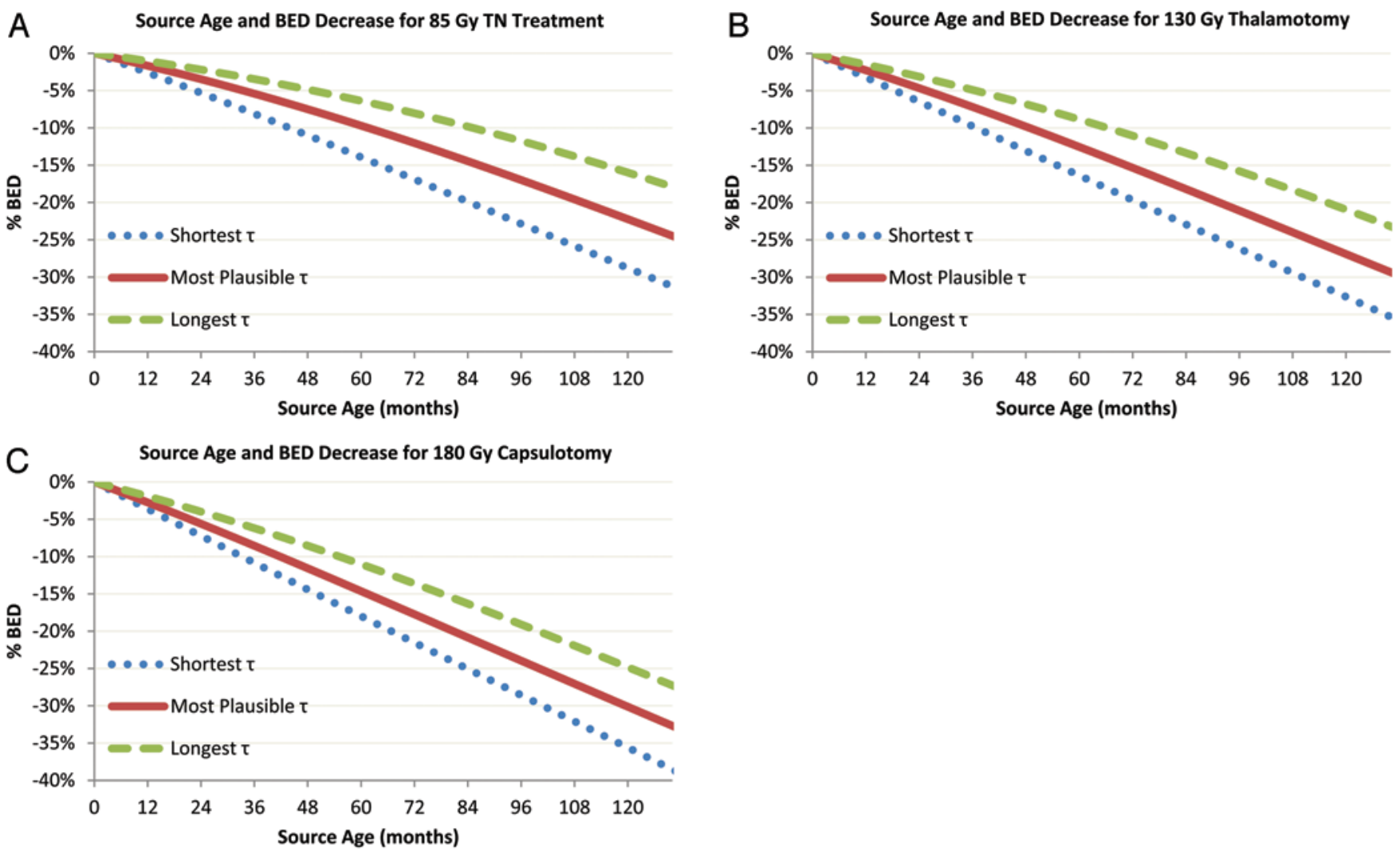

FIG. 1. Changes in BED with cobalt-60 source age in functional GKRS for different DNA repair halftime estimates for trigeminal nerve ablation for TN (A), thalamotomy (B), and capsulotomy $(C)$. $\tau$ represents the biphasic DNA repair halftimes based on literature estimates, with shortest $\tau$ of $\tau_{1}=0.19$ hours and $\tau_{2}=2.16$ hours, most plausible $\tau$ of $\tau_{1}=0.38$ hours and $\tau_{2}=4.1$ hours, and longest $\tau$ of $\tau_{1}=0.70$ hours and $\tau_{2}=6.4$ hours

tors, prescription dose, target volume and location, and radiosurgical technique. Therefore, depending on these additional factors, the impact of our model's predicted BED change on toxicity will likely vary. For example, given the proximity of the thalamus to the internal capsule and brainstem, the predicted BED increase of $15.6 \%$ with source replacement for GKRS-based thalamotomy may be of greater consequence than the $11.7 \%$ increase for TN management.

In several studies, results from variations in the LQ model have indicated that irradiation time is important in predicting the biological responses., ${ }^{711,18,23}$ Fowler et al. assumed DNA repair times similar to those used in the present study and predicted a significant correlation of BED with dose rate and irradiation time for fraction sizes commonly applied in clinical practice. ${ }^{11}$ Furthermore, some authors have proposed to give particular attention to BED variation in radiation treatments lasting more than 30 minutes per fraction. ${ }^{11}$ Millar et al., investigating the BED of GKRS in the treatment of vestibular schwannoma, reported that irradiation time was important to consider in determining the effect of a particular prescription dose. ${ }^{18}$ Our study built on these previous studies by applying a similar model to functional GKRS procedures, and its results highlight the particular risk of neglecting source age, dose rate, and treatment time for these uniquely high-dose procedures.
Only a few studies have assessed clinical end points relating to cobalt-60 source age and source replacement, which can cost between $\$ 150,000$ and $\$ 1,000,000{ }^{8,10}$ One study of GKRS for TN analyzed cobalt-60 source age and its relationship with clinical efficacy and reported that a newer source resulted in better efficacy, ${ }^{17}$ but the authors of another study observed no such correlation. ${ }^{2}$ One study of GKRS-based thalamotomy for tremor found that use of a new cobalt- 60 source decreases the time to tremor improvement. ${ }^{20}$ To our knowledge, no studies have specifically addressed the relationship between cobalt-60 source age and toxicity, and our analysis emphasizes that more study is needed to fully elucidate this relationship.

Although the LQ model remains the most widely accepted and adopted mathematical algorithm to predict BED in radiotherapy, its accuracy in predicting the BED of large fraction sizes is controversial..$^{3,13,25}$ Furthermore, the application of LQ-derived BEDs in extremely highdose treatments, such as functional GKRS, has not been validated. However, the LQ approach remains the most practical and accepted model for predicting radiationinduced tissue damage, and several studies have reported that this model provides a better fit to clinical data even at high radiosurgery doses of up to $50 \mathrm{~Gy} .{ }^{4,5,11,26}$ Of note, our model calculated BED at the target isocenter. Radiation doses at the periphery of the target and surrounding tissue will be substantially lower. Doses in these lower isodose 

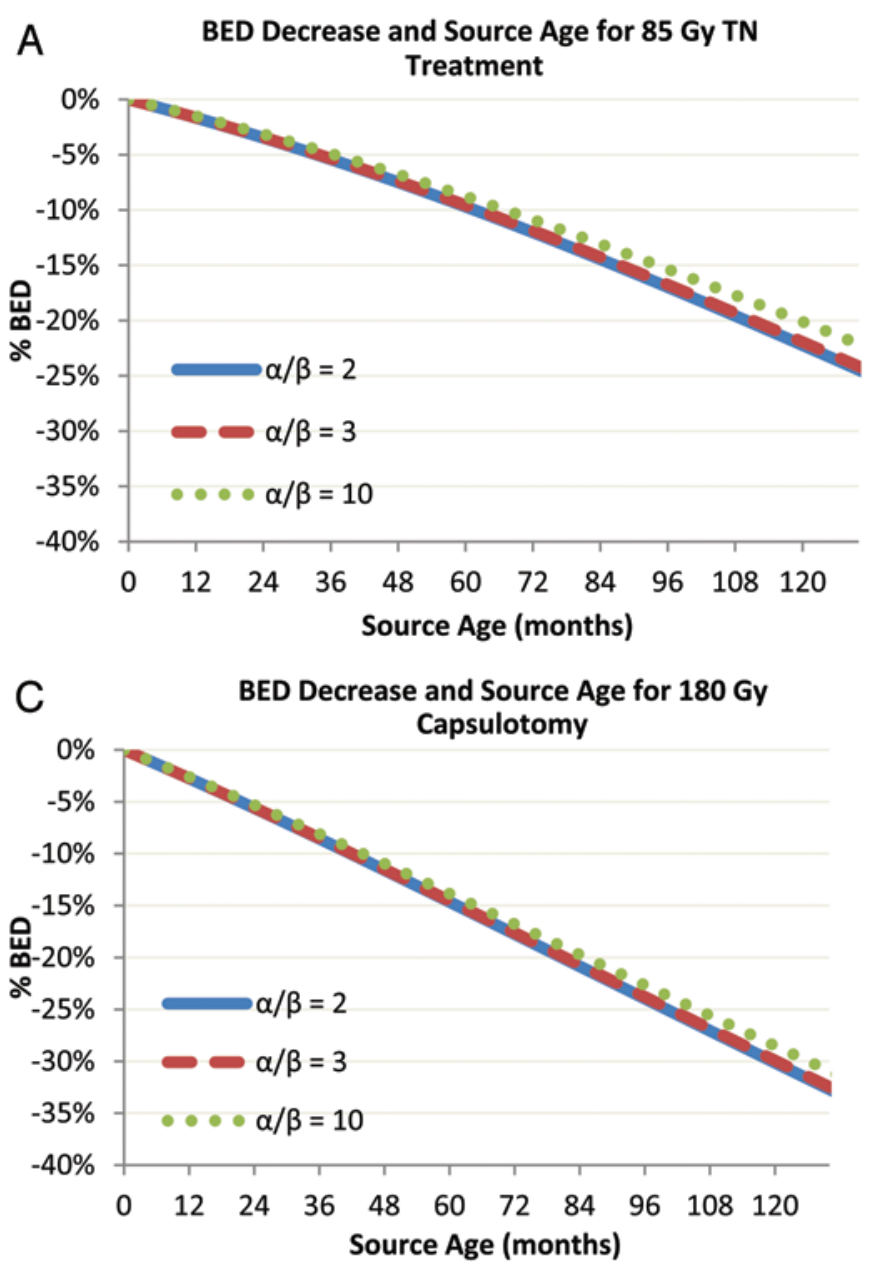

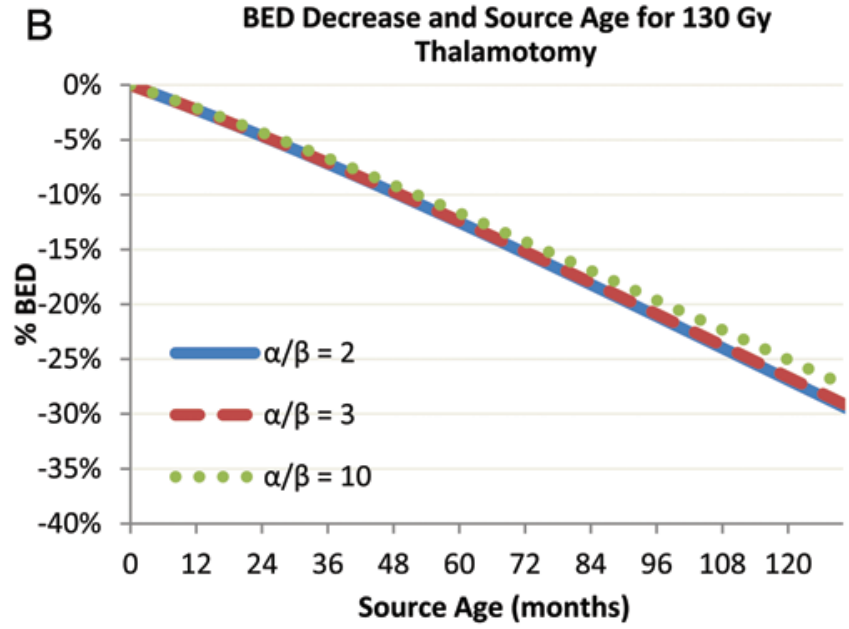

FIG. 2. Changes in BED with cobalt-60 source age in functional GKRS for different $\alpha / \beta$ values (in Gy) for TN management (A), thalamotomy (B), and capsulotomy (C).

spheres are more likely to fall within a range in which the LQ model has been validated, and BED changes in these tissues may have a clinical impact. Another possible limitation of our model is that its assumption for repair halftimes was based on rat spinal cord data. ${ }^{1,15,21}$ Although we propose that these available data based on animal experiments can be extrapolated to human CNS tissues, the repair potentials in these animal specimens likely differ from those in human tissues, and these differences may affect the broader applicability of our results.

Last, this study was theoretical in design, highlighting the need for animal and clinical studies using toxicity, efficacy, radiographic, and histopathological end points to characterize the effect of cobalt-60 source age on BED and clinical outcomes in patients. Validation of these results in clinical settings might encourage adjustment of the prescription dose according to cobalt- 60 source age and motivate consideration of other techniques, such as selective collimation, to alter dose rate and irradiation time.

\section{Conclusions}

The results of the present study indicate that after replacement of an old cobalt- 60 source, use of a new co- balt-60 source substantially increases the predicted BED for functional GKRS treatments, despite identical prescription doses. Source age, dose rate, and treatment time should all be considered in the study of outcomes of highdose functional GKRS treatments. Additional studies are needed to determine how this increase in BED contributes to toxicity to functional tissues and to evaluate whether technical adjustments should be made to reduce the radiation dose rate or prescription doses in functional GKRS with newer cobalt- 60 sources.

\section{References}

1. Ang KK, Jiang GL, Guttenberger R, Thames HD, Stephens $\mathrm{LC}$, Smith CD, et al: Impact of spinal cord repair kinetics on the practice of altered fractionation schedules. Radiother Oncol 25:287-294, 1992

2. Arai Y, Kano H, Lunsford LD, Novotny J Jr, Niranjan A, Flickinger JC, et al: Does the Gamma Knife dose rate affect outcomes in radiosurgery for trigeminal neuralgia? J Neurosurg 113 Suppl:168-171, 2010

3. Brenner DJ: The linear-quadratic model is an appropriate methodology for determining isoeffective doses at large doses per fraction. Semin Radiat Oncol 18:234-239, 2008

4. Brown JM, Brenner DJ, Carlson DJ: Dose escalation, not 
"new biology," can account for the efficacy of stereotactic body radiation therapy with non-small cell lung cancer. Int J Radiat Oncol Biol Phys 85:1159-1160, 2013

5. Brown JM, Carlson DJ, Brenner DJ: The tumor radiobiology of SRS and SBRT: are more than the 5 Rs involved? Int J Radiat Oncol Biol Phys 88:254-262, 2014

6. Campbell AM, Glover J, Chiang VLS, Gerrard J, Yu JB: Gamma Knife stereotactic radiosurgical thalamotomy for intractable tremor: a systematic review of the literature. Radiother Oncol 114:296-301, 2015

7. Carlson DJ, Stewart RD, Li XA, Jennings K, Wang JZ, Guerrero $\mathrm{M}$ : Comparison of in vitro and in vivo $\alpha / \beta$ ratios for prostate cancer. Phys Med Biol 49:4477-4491, 2004

8. Committee on Radiation Source Use and Radiation, Nuclear and Radiation Studies, Division on Earth and Life Studies, National Research Council: Radiation Source Use and Replacement: Abbreviated Version. Washington, DC: National Academies Press, 2008

9. Dale RG: The application of the linear-quadratic dose-effect equation to fractionated and protracted radiotherapy. Br J Radiol 58:515-528, 1985

10. Epstein ME, Lindquist C: Cost accounting the Gamma Knife. Stereotact Funct Neurosurg 61 (Suppl 1):6-10, 1993

11. Fowler JF, Welsh JS, Howard SP: Loss of biological effect in prolonged fraction delivery. Int J Radiat Oncol Biol Phys 59:242-249, 2004

12. Higuchi Y, Matsuda S, Serizawa T: Gamma Knife radiosurgery in movement disorders: indications and limitations. Mov Disord [epub ahead of print], 2016

13. Kirkpatrick JP, Meyer JJ, Marks LB: The linear-quadratic model is inappropriate to model high dose per fraction effects in radiosurgery. Semin Radiat Oncol 18:240-243, 2008

14. Kooshkabadi A, Lunsford LD, Tonetti D, Flickinger JC, Kondziolka D: Gamma Knife thalamotomy for tremor in the magnetic resonance imaging era. J Neurosurg 118:713-718, 2013

15. Landuyt W, Fowler J, Ruifrok A, Stüben G, van der Kogel A, van der Schueren E: Kinetics of repair in the spinal cord of the rat. Radiother Oncol 45:55-62, 1997

16. Lea DE: A theory of the action of radiations on biological materials capable of recovery, Part 1. Br J Radiol 11:489497, 1938

17. Lee JYK, Sandhu S, Miller D, Solberg T, Dorsey JF, AlonsoBasanta M: Higher dose rate Gamma Knife radiosurgery may provide earlier and longer-lasting pain relief for patients with trigeminal neuralgia. J Neurosurg 123:961-968, 2015

18. Millar WT, Hopewell JW, Paddick I, Lindquist C, Nordströn $\mathrm{H}$, Lidberg P, et al: The role of the concept of biologically effective dose (BED) in treatment planning in radiosurgery. Phys Med 31:627-633, 2015

19. Ohye C, Shibazaki T, Hirato M, Inoue H, Andou Y: Gamma thalamotomy for parkinsonian and other kinds of tremor. Stereotact Funct Neurosurg 66 (Suppl 1):333-342, 1996

20. Ohye C, Shibazaki T, Sato S: Gamma Knife thalamotomy for movement disorders: evaluation of the thalamic lesion and clinical results. J Neurosurg 102 Suppl:234-240, 2005

21. Pop LAM, Millar WT, van der Plas M, van der Kogel AJ: Radiation tolerance of rat spinal cord to pulsed dose rate (PDR-) brachytherapy: the impact of differences in temporal dose distribution. Radiother Oncol 55:301-315, 2000

22. Régis J: Gamma Knife for functional diseases. Neurotherapeutics 11:583-592, 2014

23. Sachs RK, Hahnfeld P, Brenner DJ: The link between lowLET dose-response relations and the underlying kinetics of damage production/repair/misrepair. Int J Radiat Biol 72:351-374, 1997

24. Sheehan J, Pan HC, Stroila M, Steiner L: Gamma Knife surgery for trigeminal neuralgia: outcomes and prognostic factors. J Neurosurg 111 Suppl:434-441, 2009

25. Shibamoto Y, Otsuka S, Iwata H, Sugie C, Ogino H, Tomita $\mathrm{N}$ : Radiobiological evaluation of the radiation dose as used in high-precision radiotherapy: effect of prolonged delivery time and applicability of the linear-quadratic model. J Radiat Res (Tokyo) 53:1-9, 2012

26. Shuryak I, Carlson DJ, Brown JM, Brenner DJ: High-dose and fractionation effects in stereotactic radiation therapy: Analysis of tumor control data from 2965 patients. Radiother Oncol 115:327-334, 2015

27. Young RF, Li F, Vermeulen S, Meier R: Gamma Knife thalamotomy for treatment of essential tremor: long-term results. J Neurosurg 112:1311-1317, 2010

\section{Disclosures}

The authors report no conflict of interest concerning the materials or methods used in this study or the findings specified in this paper.

\section{Author Contributions}

Conception and design: Kann, Yu, Loiselle, Carlson. Acquisition of data: Kann, Yu, Bond, Gerrard, Carlson. Analysis and interpretation of data: Kann, Yu, Stahl, Bond, Carlson. Drafting the article: Kann, Yu, Chiang, Carlson. Critically revising the article: all authors. Reviewed submitted version of manuscript: all authors. Approved the final version of the manuscript on behalf of all authors: Kann. Administrative/technical/material support: Kann, Carlson. Study supervision: Yu, Loiselle, Chiang, Bindra, Gerrard, Carlson.

\section{Supplemental Information \\ Previous Presentations}

Portions of this work were presented as a talk at the 18th Leksell Gamma Knife Society Meeting in Amsterdam, May 15-19, 2016.

\section{Correspondence}

Benjamin H. Kann, Yale University School of Medicine, Therapeutic Radiology, 135 Park St., LL509, New Haven, CT 06519. email: benjamin.kann@yale.edu. 